\title{
SARS-CoV-2 and HIV: Convergence of Two Pandemics in the Caribbean
}

\author{
Robert Paulino-Ramirez ${ }^{1}$
}

\section{ABSTRACT}

This article describes the social, biological, and programmatic interactions between HIV and SARS-CoV-2 co-infections in The Caribbean region. The country islands represent the second most affected region by HIVIAIDS after Sub-Saharan Africa. After the first confirmed COVID-19 cases in the region, it is necessary to reinvent the programmatic interventions designed to eliminate HIV transmission, and implement innovative interventions that are not yet currently available like PrEP, HIV self-testing, and multiple medication dispensing. COVID-19 pandemic offers an opportunity to governments, community-based organizations, and international partners to maintain the HIV care continuum, with particular efforts made to ensure timely access to, and to avoid disruption of routinely HIV services.

SARS-CoV-2 has spread voraciously across the world since the first reports in Wuhan City in December 2019 and affecting to the date more than 29 million people worldwide [1]. In contrast, a much older HIV pandemic that has been endemically surviving all public health efforts since the $80 \mathrm{~s}$ affecting near 38 million people worldwide [2], Putting those already immunologically challenged individuals at risk with coronavirus disease (COVID-19). The Caribbean has been the second largely affected region by HIV/AIDS after sub-Saharan Africa, with more than 340,000 people diagnosed by 2019 , representing $1.2 \%$ prevalence in contrast with a $0.4 \%$ in the rest of Latin American countries [2]. As of September 2020, a total of 191,931 cumulative COVID-19 cases has been reported from the Caribbean [3].

Despite the impressive progress made in Latin America in increasing the number of people knowing their HIV status, to almost $80 \%$, the percentage of suppressed viral loads is still below the global average. In contrast, the Caribbean countries are only achieving a $72 \%$ of awareness of HIV status, and only $77 \%$ of them enrolled into HIV treatment [2]. By the end of 2019, nearly $90 \%$ of new infections occurred in four Caribbean countries: Dominican Republic,
Haiti, Jamaica, and Cuba, while $87 \%$ of deaths from AIDSdefining illnesses occurred in the Dominican Republic and Haiti [2]. However, Cuba and the Dominican Republic are profiling better on the first UNAIDS 90: percentage of people living with HIV that know their status, with $83 \%$ and $82 \%$, respectively.

With the COVID-19 pandemic across the region, implementation of quarantine, social distancing, and community containment measures have affected the HIV routine testing, service provision, preventive interventions to reduce transmission, and personal and psychological challenges to people living with HIV (PLWH).

UNAIDS set this year as the 90-90-90 goal country evaluations, and envisioning an increase to $95 \%$ to the HIV cascade of care indicators. Firstly, to achieve the 90 goal testing to initiate HIV care, some countries across the Caribbean has been implementing HIV self-testing as an engagement method in those hard-to-reach population. However, only The Bahamas and Jamaica has programmatic guidelines for it use, the remaining countries, including the Dominican Republic, are yet to document their use or implementing this method to expand testing to people from

${ }^{1}$ Instituto de Medicina Tropical \& Salud Global. Universidad Iberoamericana, Santo Domingo, Dominican Republic

Como citar este artigo / How to cite this article

Paulino Ramirez R. SARS-CoV-2 and HIV: Convergence of Two Pandemics in the Caribbean. InterAm J Med Health 2020;3: e202003057. 
populations, due to a concentrated epidemic among men who have sex with men, transgender populations, and migrants.

Second, is still very limited documented the experiences between viral and immune response interactions between HIV and SARS-CoV-2; in a case report series from Spain, no attributable benefits associated to specific antiretroviral therapy (ART) [4], pointing the importance on immune status, and ART use; in a separate case series from 33 patients from Germany [5] the authors reported a $9 \%$ case mortality rate, with no correlation between use or not use of ART, and no excess mortality or morbidity observed. Unfortunately, no case reports or experiences from Latin America or the region have yet published considering the socio-economical and intervention contexts compared with European countries.

Host immune responses to SARS-CoV-2 linked to an exhaustion of T lymphocytes [6, 7]. Despite the limited information from in-vivo studies, many articles have been proposing an inflammatory mechanism associated with regulated and dysregulated responses. In addition to local pulmonary damage, cytokine storms are inducing systemic effects that lead to septic shock and multi-organ failure. These dysfunctional immune responses are associated with older people (aged over 60 years) and people with comorbidities, provoking an altered dendritic cell maturation and migration to the lymphoid organs, and thereby $T$ cell defective activation8. Coronavirus-specific $T$ cells are involved in viral elimination and disease progression, but also crucial in vaccine development and evaluation.

Indicators mentioned above, reflects on the importance of proposing an individuals' risks categorization based on their immune status at the moment of COVID-19 diagnosis: a) Those with suppressed HIV viral load and CD4+ T cell count $>350$ cells $/ \mathrm{mL}$; b) Those with CD4+ T cell count $<350 \mathrm{cell} / \mathrm{mL}$, and suppressed HIV viral load, and c) Newly diagnosed individuals with unknown CD4+ T cell and HIV viral load status.

Despite there is no consensus on protease inhibitors (PIs), use benefits [9], nor effective inhibition of 3-chymotrypsin-like and papain-like proteases of SARSCoV-2. Many PLWH are receiving ART regimens based on integrase inhibitors (INSTIS), like Dolutegravir, rather than Pls. Therefore, a possible protection from the use of HIV PIs does not seem to be a rational explanation for a reduced disease risk progression, yet still to be evaluated in longitudinal studies. However, to the present, there is no evidence to suggest that there is an increased risk of infection and severity of illness for people living with HIV.
Some publications described that during the SARS and MERS outbreaks, there were only a few case reports of mild disease among people living with HIV [10].

Third, in this pandemic context, early ART initiation is confronting the reality of busy hospitals and saturated personnel treating patients with COVID-19, delaying and deterring early initiation, or only by PLWH scared to visit hospitals dedicated to COVID-19 attention. In the Dominican Republic, first mortality associated to COVID-19 was reported in an HIV positive woman with tuberculosis co-infection, ringing concerns on PLWH, affecting clinical appointments, and calling attention to policymakers on another respiratory illness that have a long-living synergy with HIV in the tropics [11].

As many countries have been implementing city lockdowns or traffic controls, hospital visits could be limited or costly to afford when public transportation is not available.

Furthermore, other preventive strategies like preexposure prophylaxis (PrEP) might be affected, this yet limited intervention across the island nations, PrEP implementation programs has been running in the Caribbean, examples from the Dominican Republic, and Cuba, has galvanized the interest from other islands to start this preventive measure focus on MSM and Transgender individuals12. Gender-based violence interventions, mother-to-child transmission preventive programs, condom distributions, among others, and the redirecting funding from HIV programs to be dedicated to COVID-19 public health interventions, would affect resources dedicated to HIV care and continuum.

Finally, the COVID-19 pandemic represents an unprecedented public health emergency, that affects cross-sectionally all health care services, and this includes HIV, with previous barriers of access experienced by marginalized communities, but also provides an opportunity for decision-makers, and stakeholders to reinvent the local responses in the aim to continue to eliminate HIV/ AIDS from the global agenda. As COVID-19 continues to spread worldwide, many communities are facing the risk of SARS-COV-2 infection and barriers in maintaining the HIV care continuum. This situation could be worsening in places with weak health-care systems. As in the early ages of HIV response, community-based organizations, local governments, and international collaboration should work together to strengthen the HIV care continuum during the COVID-19 pandemic, with efforts made to ensure access to, and to avoid disruption of routinely HIV services, with early reporting, and scientific communications of frontline 
providers from the region. SARS-CoV-2 epidemiological patterns seems that to interact with HIV in a continuum; maybe the next guidelines would include management, and preventive measures for most-at-risk populations living with HIV.

\section{REFERENCES}

1. Johns Hopkins University, Coronavirus Resource: https://coronavirus.jhu.edu/map. html . Accessed on September 14, 2020.

2. UNAIDS 2019 Global Report: https://www.unaids. org/sites/default/files/media_asset/2019-UNAIDSdata_en.pdf . Accessed on September 14, 2020.

3. Panamerican Health Organization COVID-19 Report in the Americas: http://ais.paho.org/phip/viz/ COVID19Table.asp . Accessed on May 14, 2020.

4. Blanco JL, Ambrosioni J, Garcia F, Martinez E. COVID-19 in patients with HIV: Clinical case series. The Lancet HIV. 2020; 7. http://dx.doi.org/10.1016/S2352-3018(20)30111-9

5. HärterG, SpinnerC.D., Roider J, BickelM, Krznaricl, Grunwald S, Schabaz F, et al. 2020. COVID-19 in people living with human immunodeficiency virus: a case series of 33 patients. Infection. http://dx.doi.org/10.1007/s15010-020-01438-z

6. Tay, MZ, Poh CM, Rénia L. The trinity of COVID-19: immunity, inflammation and intervention. Nat Rev Immunol. 2020 . http://dx.doi.org/10.1038/s41577-020-0311-8

7. Moon C. 2020. Fighting COVID-19 exhausts $T$ cells. Nat Rev Immunol. 2020; 20(5):277. http://dx.doi.org/10.1038/s41577-020-0304-7

8. Diao B. 2020. Reduction and functional exhaustion of T cells in patients with coronavirus disease 2019 (COVID-19). Medrxiv. 2020. http://dx.doi.org/10.1101/2020.02.18.20024364

9. WHO. Q\&A on COVID-19, HIV and antiretrovirals. March 24, 2020. https:// www.who. int news-room/q-a-detail/q-a-on-covid-19-hiv-andantiretrovirals Accessed on Septmeber 14, 2020.
10. Sarah S, Abdulwahab A, Raed S, Adnan M. 2015. Successful recovery of MERS CoV pneumonia in a patient with acquired immunodeficiency syndrome: A case report. J Clin Virol. 2015; 62: 6971. http://dx.doi.org/10.1016/j.jcv.2014.11.030

11. Dominican Republic Ministry of Health, COVID-19 Special Epidemiological Bulletins \#2, https://www.msp.gob. do/web/wp-content/uploads/2020/04/Bolet\%C3\%ADnEspecial-01.pdf . Accessed on September 14, 2020.

12. PrEP Watch. https://www.prepwatch. org/country/dominican-republic/ 